\title{
Solar wind electron interaction with the dayside lunar surface and crustal magnetic fields: Evidence for precursor effects
}

\author{
J. S. Halekas ${ }^{1,2}$, A. Poppe ${ }^{1,2}$, G. T. Delory ${ }^{1,2}$, W. M. Farrell ${ }^{4,2}$, and M. Horányi ${ }^{3,2}$ \\ ${ }^{1}$ Space Sciences Laboratory, 7 Gauss Way, University of California, Berkeley, CA 94720, USA \\ ${ }^{2}$ NASA's Lunar Science Institute, NASA Ames Research Center, Moffett Field, CA 94035, USA \\ ${ }^{3}$ Laboratory for Atmospheric and Space Physics, and Department of Physics, University of Colorado, Boulder, CO 80309, USA \\ ${ }^{4}$ NASA Goddard Space Flight Center, Greenbelt, MD 20771, USA
}

(Received January 18, 2011; Revised March 10, 2011; Accepted March 22, 2011; Online published March 8, 2012)

\begin{abstract}
Electron distributions measured by Lunar Prospector above the dayside lunar surface in the solar wind often have an energy dependent loss cone, inconsistent with adiabatic magnetic reflection. Energy dependent reflection suggests the presence of downward parallel electric fields below the spacecraft, possibly indicating the presence of a standing electrostatic structure. Many electron distributions contain apparent low energy $(<100 \mathrm{eV})$ upwardgoing conics ( $58 \%$ of the time) and beams (12\% of the time), primarily in regions with non-zero crustal magnetic fields, implying the presence of parallel electric fields and/or wave-particle interactions below the spacecraft. Some, but not all, of the observed energy dependence comes from the energy gained during reflection from a moving obstacle; correctly characterizing electron reflection requires the use of the proper reference frame. Nonadiabatic reflection may also play a role, but cannot fully explain observations. In cases with upward-going beams, we observe partial isotropization of incoming solar wind electrons, possibly indicating streaming and/or whistler instabilities. The Moon may therefore influence solar wind plasma well upstream from its surface. Magnetic anomaly interactions and/or non-monotonic near surface potentials provide the most likely candidates to produce the observed precursor effects, which may help ensure quasi-neutrality upstream from the Moon.
\end{abstract}

Key words: Moon, solar wind, precursor effects, wave-particle interactions.

\section{Introduction}

The Moon, with only a tenuous atmosphere and no global magnetic field, seemingly should present a relatively simple obstacle to the solar wind flow, with the expected Moon-solar wind interaction dominated by the absorption of plasma at the surface and the formation of a downstream plasma wake. However, the lunar interaction in fact has a number of surprisingly complicated aspects (Halekas et al., 2010b), some with effects that may propagate well upstream from the lunar surface. In this paper, we investigate the effects of the Moon-solar wind interaction on electron distributions measured above the dayside lunar surface.

Since the Apollo era, we have recognized that the smallscale lunar crustal magnetic fields can perturb the incoming solar wind flow and affect the characteristics of plasma reaching the surface (Neugebauer et al., 1972; Goldstein, 1974; Clay et al., 1975). More recently, measurements from Lunar Prospector, Kaguya, and Chandrayaan have shown that some of the stronger magnetic anomalies can at least partially shield the lunar surface from the solar wind flow (Lin et al., 1998; Halekas et al., 2008a; Saito et al., 2010; Wieser et al., 2010). The magnetic anomaly interaction can also compress and/or shock the incoming solar wind (Lin et al., 1998), generate plasma waves (Halekas et al.,

Copyright (C) The Society of Geomagnetism and Earth, Planetary and Space Sciences (SGEPSS); The Seismological Society of Japan; The Volcanological Society of Japan; The Geodetic Society of Japan; The Japanese Society for Planetary Sciences; TERRAPUB.

doi: $10.5047 /$ eps.2011.03.008
2006), and heat the incoming solar wind electron population (Halekas et al., 2008b; Saito et al., 2010).

Even outside of the influence of crustal fields, we now know that part of the solar wind ion population reflects from the surface, in both ion (Saito et al., 2008) and more often in neutral form (Wieser et al., 2009; McComas et al., 2009). The dayside lunar surface also provides a source of low energy photoelectrons produced by solar photons and secondary electrons produced by both electron and ion impact, as well as reflected and backscattered primary electrons (Feuerbacher et al., 1972; Willis et al., 1973; Whipple, 1981; Horányi et al., 1998).

These various populations of ions and electrons contribute to charge the surface to a floating electrostatic potential. Simple probe theory treats this as a current balance problem (e.g. Manka, 1973), and most measurements to date suggest small positive dayside lunar surface potentials seemingly consistent with a balance between photoemission and incident solar wind electron thermal currents (Goldstein, 1974; Freeman and Ibrahim, 1975; Halekas et al., 2008c). However, some theory and simulation results suggest that space charge may also play a role in the interaction, possibly leading to non-monotonic potential structures above the lunar surface (Guernsey and Fu, 1970; Nitter et al., 1998; Poppe and Horányi, 2010; Poppe et al., 2011).

In the simplest view, a plasma sheath forms above a surface to screen the charged surface, thereby minimizing any feedback effects on the ambient plasma. Therefore, one would naively expect little modification of the incoming 
solar wind plasma as a result of the proximity of the lunar surface. However, the absorption of part of the electron population and resulting depletion of solar wind electrons on flux tubes connected to the Moon, as well as the feedback of ions and electrons reflected from and/or produced at the surface, implies that the presence of the Moon must affect the incoming solar wind to some degree.

In this work, we investigate the characteristics of electron distributions observed above the dayside lunar surface in the solar wind by Lunar Prospector, in order to characterize the effects of the solar wind interaction with the lunar surface and crustal anomalies. We find unexpected features in the upward-going electron populations, and occasionally also in downward-going populations, indicative of significant effects on solar wind electrons above the dayside surface.

\section{Electron Observations}

\subsection{Lunar Prospector Electron Reflectometer data}

This study utilizes data from the Lunar Prospector Electron Reflectometer (LP ER) to characterize electron distributions above the dayside lunar surface. The ER employed a top-hat electrostatic analyzer design with a $360^{\circ}$ planar field of view oriented perpendicular to the spin plane, enabling a measurement of the full 3-D electron distribution every half-spin $(2.5 \mathrm{~s})$. The ER had an intrinsic energy resolution of $\Delta E / E=\sim 0.25$, but the onboard processor summed adjacent energy bins together, resulting in an effective $\Delta E / E=\sim 0.5$. For all observations analyzed in this paper, the instrument measured electrons in 15 energy bins with center energies logarithmically spaced from $38 \mathrm{eV}$ to $17 \mathrm{keV}$. Though the ER only had sufficient telemetry to send back full 3-D measurements with an 80 s cadence (corresponding to $120 \mathrm{~km}$ spatial separation), each integration lasted only $2.5 \mathrm{~s}$, ensuring an intrinsic spatial resolution for each individual measurement of a few $\mathrm{km}$.

We applied two basic corrections to all observations used in this study. First, we corrected measured count rates for relative anode sensitivity, eliminating any internal instrumental variation in counting efficiency. Second, we shifted electron distributions in energy to take into account the floating potential of the spacecraft. Since we cannot always determine this potential reliably in the solar wind (due to the limited energy range), we use a single assumed value of +10 volts, consistent with the average expected potential for LP in the solar wind (Halekas et al., 2008c). In actuality, spacecraft potential changes with plasma conditions, but in sunlight in the solar wind it remains in a narrow range. Small variations in this parameter could introduce slight errors in the frame transformations described below, but should not significantly affect any of our results.

\subsection{Data selection}

We selected observations best suited for investigating the dayside Moon-solar wind interaction. To minimize the local effects of crustal magnetic field interactions (as opposed to those below the spacecraft) we used data only from the first mission year (1998), when LP maintained a $\sim 100 \mathrm{~km}$ altitude, well above strong crustal fields (the crustal field signal at $\sim 100 \mathrm{~km}$ is $<2 \mathrm{nT}$ ). We chose time periods with the Wind spacecraft upstream from the Moon and the Earth and outside of the terrestrial foreshock, so it could act as an upstream monitor. We picked orbits with good solar zenith angle coverage (LP not in a dawn-dusk orbit), and chose only the portions of those orbits with LP and its magnetic foot-point both in sunlight. Next, we removed active events (e.g. solar energetic particles), leaving five epochs in March, May, August, September, and October of 1998. Finally, we removed data points for which the magnetic field magnitude measured at Wind (time-shifted by solar wind travel time) did not match that measured at LP to within $20 \%$, thus minimizing the effects of solar wind inhomogeneities and local field compression above strong lunar crustal fields. Application of these selection criteria resulted in a data set of 8625 observations. Finally, for ease of analysis, we selected 4902 of those data points for which the electron loss cone was well fit by an adiabatic model for combined magnetic and electrostatic reflection (this includes end-member cases with no magnetic and/or no electrostatic reflection) (Halekas et al., 2008c). This final down-selection removes data points over the strongest magnetic anomalies without a measurable loss cone, times with tenuous magnetic connection to the surface, and poor/noisy data. This greatly facilitated semi-automated analysis, but did not significantly affect the relevant properties of the data set. All features discussed subsequently remain equally apparent before and after this down-selection.

\subsection{Electron distribution functions}

We calculate the median electron distribution, as a function of parallel and perpendicular velocity (applying all the corrections described in Section 2.1), in three different reference frames, and display the results in Fig. 1. To calculate this median distribution, we calculate individual velocity distributions for each observation time, and then calculate overall medians at each velocity point (we use medians rather than means to avoid outlier effects). Since distribution function varies over many orders of magnitude, small differences can prove difficult to visually identify. Therefore, we also display median distributions normalized by the angle-averaged energy dependence, in order to highlight angular variations. In all cases, we first transform reference frames for each individual observation, based on the parameters appropriate for that time, and then calculate the median distribution.

The first reference frame, the base frame for our analysis, is the solar wind frame. In this frame, electrons gyrate around the interplanetary magnetic field (IMF), feeling no solar wind convection electric field, and should therefore have gyrotropic distributions. In the solar wind frame, the lunar surface represents a moving obstacle, generally oriented obliquely to its motion, so any reflection process introduces an extra velocity to the reflected electrons, with both parallel and perpendicular components.

In the second frame, the Moon rest frame, the Moon obviously represents a stationary obstacle. However, in this frame the electrons see a non-zero convection electric field, so any reflection process analyzed in this frame involves a complicated mix of particle drifts, with both parallel and perpendicular effects.

The third frame, the most useful for considering reflection from a moving obstacle, is the deHoffman-Teller (dHT) frame (deHoffman and Teller, 1950). This frame has a 

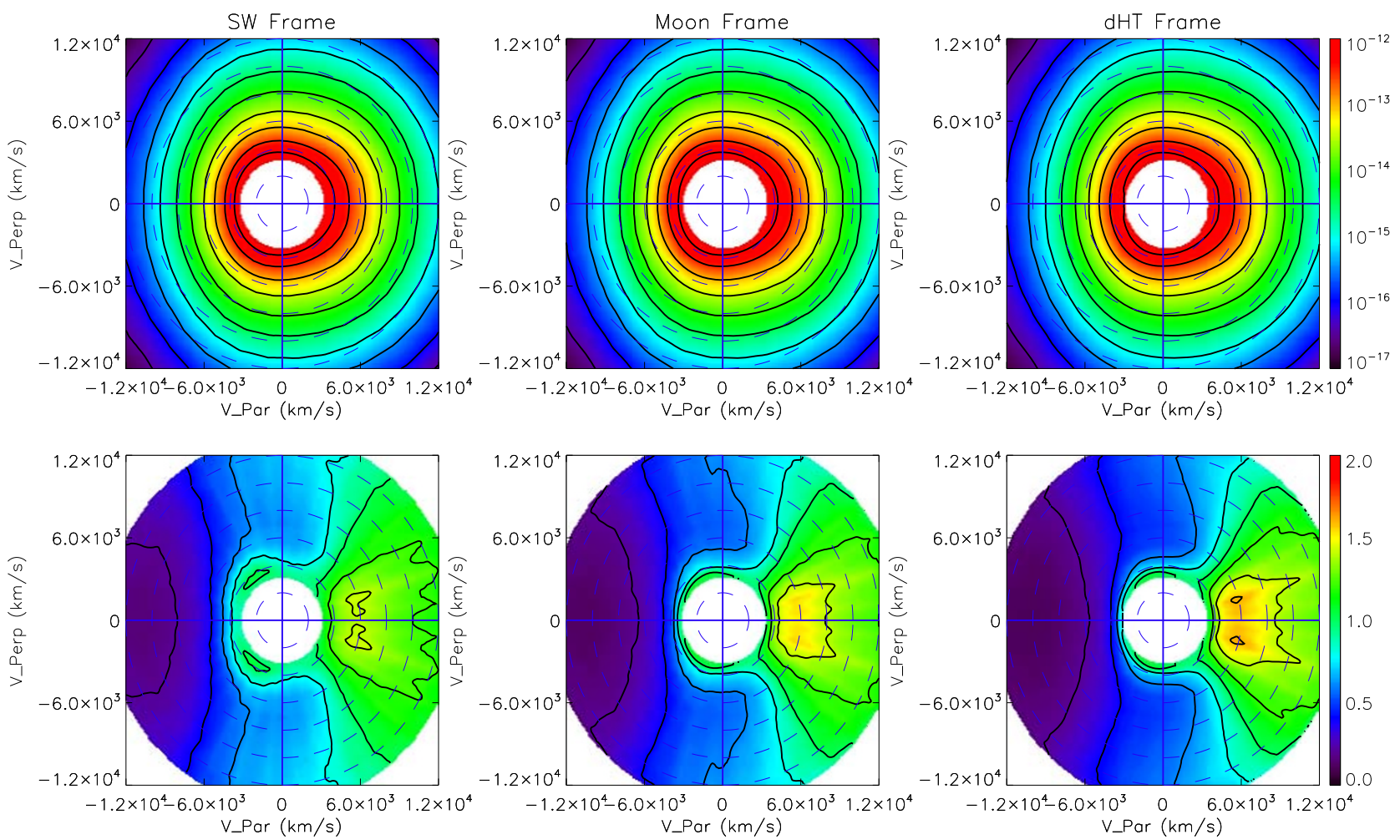

Fig. 1. Top three panels show median electron distribution functions $\left[(\mathrm{km} / \mathrm{s})^{-3} / \mathrm{cm}^{-3}\right]$ observed at 4902 times in March-October 1998 when the Lunar Prospector spacecraft was magnetically connected to the dayside lunar surface in the solar wind and the Electron Reflectometer measured a loss cone, in solar wind, Moon, and deHoffman-Teller frames. Positive parallel velocity points downward towards the surface (along the magnetic field) and negative parallel velocity points upward. Given roughly gyrotropic distributions, we only calculate a single perpendicular velocity, but show it with both positive and negative polarity for ease of visualization. The bottom three panels show the same data, but normalized by the angle-averaged energy spectrum of the downward-going electron population. This normalization removes energy dependence, but leaves angular variations, allowing easier identification of electron anisotropies.
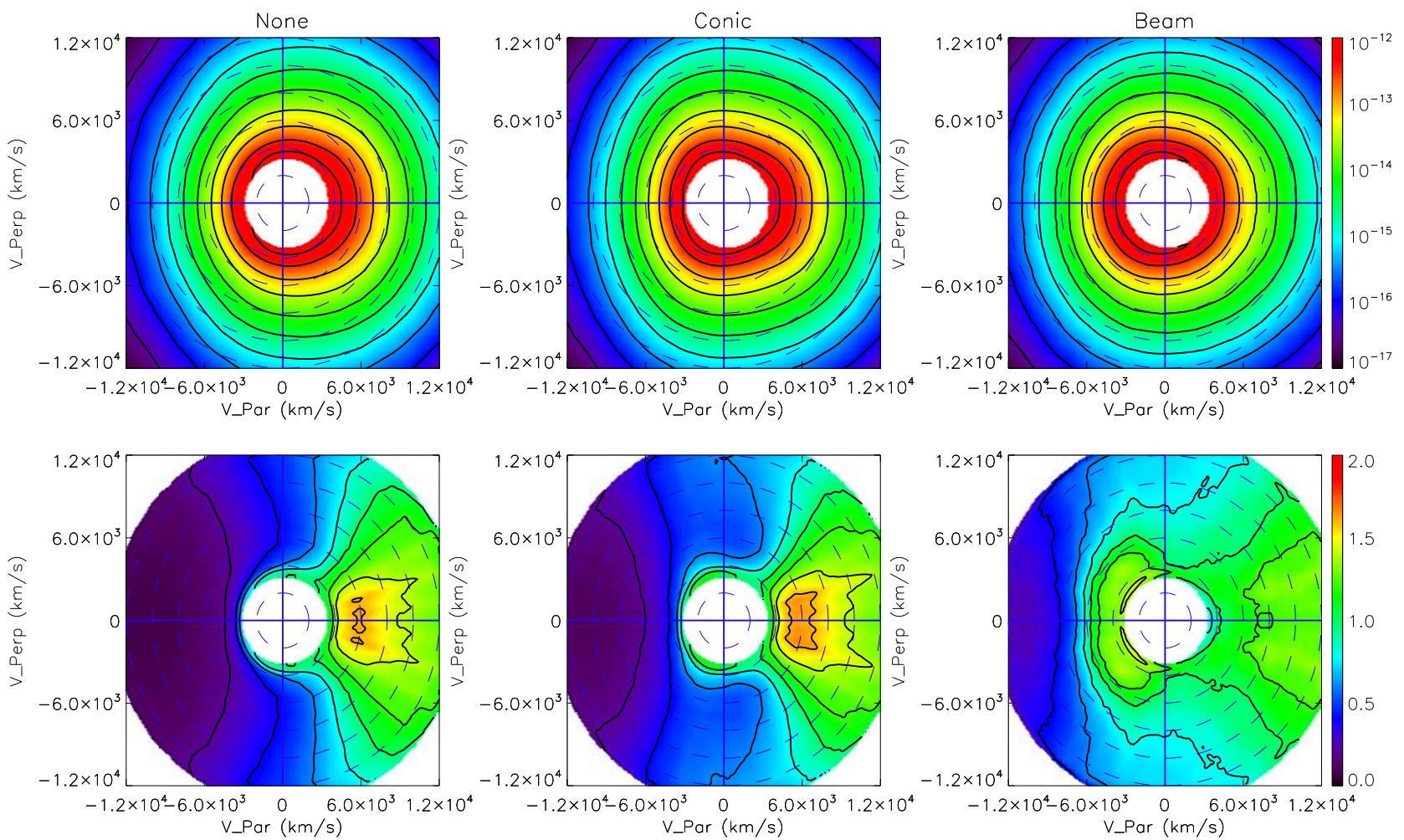

Fig. 2. Median electron distributions in the deHoffman-Teller frame for 1469 observations that have no upward-going enhancement ("None"), 2853 observations that have an apparent oblique upward-going enhancement ("Conic"), and 580 observations that have an apparent parallel upward-going flux enhancement ("Beam"), all in the same format as Fig. 1. 
transformation velocity relative to the solar wind frame, with magnitude $-\mathbf{v} \cdot \mathbf{n} /(\hat{\mathbf{B}} \cdot \mathbf{n})$, aligned with the IMF (here, $\mathbf{v}$, $\mathbf{n}$, and $\mathbf{B}$ represent the solar wind velocity, obstacle normal, and magnetic field vectors). In the dHT frame, the solar wind flows parallel to the IMF by construction, such that no convection electric field exists. Also, this frame moves purely laterally relative to the obstacle surface; therefore, given a laterally homogenous obstacle, we can treat it as stationary in the dHT frame. As a result, any adiabatic reflection process perfectly conserves energy in the dHT frame, with parallel velocities reversed and perpendicular velocities unchanged for reflecting particles. For this frame transformation, we assume that any reflection surface lies parallel to the lunar surface, at the footpoint of the field line. If the reflection surface actually has a different orientation (e.g. over crustal magnetic anomalies), or if it has lateral non-uniformities, this could result in frame transformation errors.

The resulting median velocity distribution displays a number of interesting properties in all three frames. Downward-going electrons display a clear anisotropy, with higher parallel than perpendicular temperature, typical of suprathermal solar wind electrons (i.e. the halo/strahl). Upward-going electrons, meanwhile, have an energy dependent loss cone, generally indicative of the presence of parallel electric fields below the spacecraft (Halekas et al., 2008c). Finally, upward-going electrons have enhanced perpendicular flux at low energies - this type of oblique enhancement or "conic", often observed in Earth's auroral regions, can indicate electron heating (Menietti and Burch, 1985). Note that the conic feature appears more clearly in the solar wind frame than in the Moon or dHT frames, so it results partially, but not completely, from reflection from a moving obstacle. Any physical explanation of the electron characteristics must explain both the energy dependent loss cones and the upward-going conics.

We investigated all 4902 selected electron distributions and manually classified them (in the solar wind rest frame) in terms of those with no upward-going enhancement in flux, those with an upward-going conic, and those with an upward-going beam. We display the median velocity distributions of the three resulting populations in Fig. 2, in the same format as Fig. 1, in the dHT frame. In addition, we show energy-angle spectrograms for the same three populations in the solar wind and dHT frames in Figs. 3 and 4 , normalized so that we can easily identify any departures from adiabatic reflection. In Figs. 3 and 4, any value above unity indicates an enhancement in upward-going electron flux that cannot be explained by purely adiabatic reflection of the source population in the respective velocity frame.

The first type of event, with no apparent upward-going flux enhancement (30\% of observations), does in fact display features broadly consistent with adiabatic reflection of the incoming solar wind distribution. The energy dependence of the loss cone, on average, suggests the presence of a negative potential on the order of a few tens of volts below the spacecraft, located between the spacecraft and the surface. This inferred value varies among the members of this population, but the form of the loss cone remains very consistent. Individual observations have a sharper loss cone than the median distribution presented herein (since averaging over variability inevitably "smears out" this feature), but we never observe perfectly sharp loss cones, possibly indicating the presence of processes that act to smooth out the sharp discontinuity in the electron distribution at the loss cone boundary. We find a smaller amount of energy dependence in the dHT frame, indicating that the energy dependence results partly from reflection from a moving obstacle. However, the reflection process apparently has some intrinsic energy dependence even in the dHT frame, consistent with reflection by a downward parallel electric field.

The second type of event, with an apparent upward-going conic ( $58 \%$ of observations), has a similar loss cone to the first, but with enhanced flux surrounding this loss cone. The flux enhancement consists of "wings" on the upwardgoing half of the distribution extending in the perpendicular velocity direction, suggestive of perpendicular heating of the electrons. As above, the width and depth of the observed loss cone varies among the members of this population, but the form of the loss cone and the apparent oblique flux enhancement remains very consistent in the solar wind frame. The energy dependent loss cone clearly appears in all frames, indicating a truly energy-dependent reflection process. The conic, on the other hand, appears more prominently in the solar wind frame than the dHT frame, indicating that it arises partly from reflection from a moving obstacle, as also observed in the terrestrial foreshock (Fitzenreiter et al., 1990). In essence, the conic results partially from the parallel translation in velocity space of a loss cone distribution. However, a weak conic signature also remains in the dHT frame, possibly indicating that some other heating process operates. Alternatively, this signature could result from errors in our frame transformation over crustal magnetic fields or from non-adiabatic effects resulting from the small spatial scale of the reflection region.

The third type of event, with an upward-going beam (12\% of observations), has significant differences in both the downward-going and upward-going half of the distribution. First, the downward-going solar wind electrons are more isotropic than the first two populations, indicating either different solar wind properties or modification of the incoming solar wind population as a result of lunar influences (the spatial distribution of events will argue strongly for the latter possibility). Meanwhile, the upward-going electrons again have an energy dependent loss cone, but this time consistent with a larger negative potential below the spacecraft. In addition, this population has a clear enhancement of parallel flux extending up to $\sim 100 \mathrm{eV}$, with a conic signature extending even higher in energy, possibly consistent with secondary electrons and/or photoelectrons accelerated upward from the surface (Halekas et al., 2008c). The energy of the beam and the shape of the loss cone have a high variability among the members of this population, more so than the other two populations considered above. In the dHT frame, the distribution does not change dramatically, except that the flux enhancement appears slightly more oblique, possibly indicating conic formation or perhaps parallel relaxation of the beam as a result of streaming instabilities. 

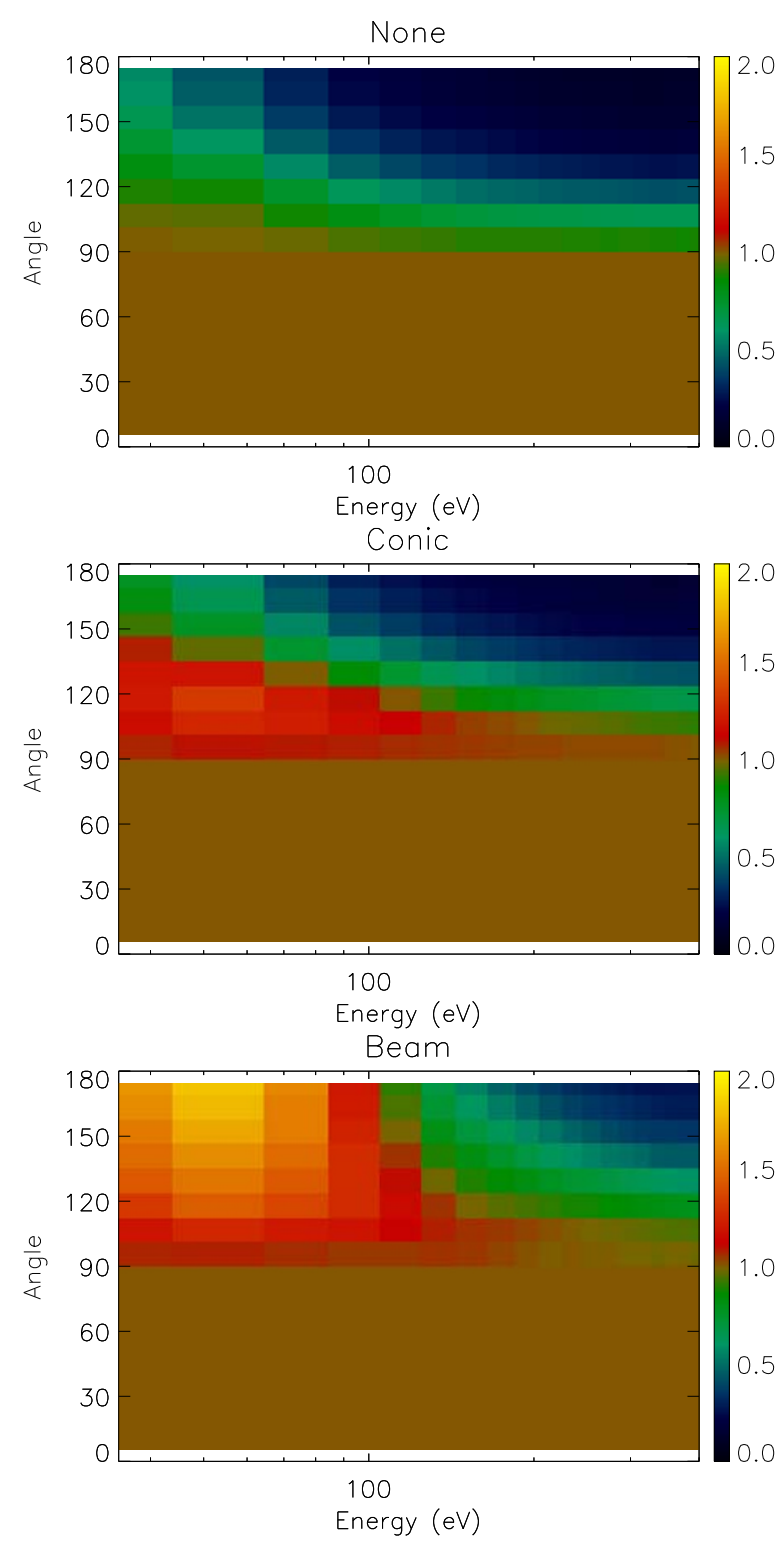

Fig. 3. Median energy-angle distributions for the same three populations in Fig. 2, in the solar wind frame, for energies of $35-400 \mathrm{eV}$. We normalize both the upward-going and downward-going halves of the electron distribution by the downward-going electron distribution such that the values for upward-going angles (90-180) show the ratio of upward-going flux at angle $180-\alpha$ to downward-going flux at the conjugate angle $\alpha$. This normalization, unlike that in the lower panels of Figs. 1 and 2, removes both the energy and angle dependence of the incoming population, allowing clear identification of departures from electron reflection expected from an adiabatic model (indicated by values above unity).

\subsection{Distribution of events}

The three populations of events described above do not have uniform spatial distributions over the surface. Rather, the type of event most commonly observed appears to vary as a function of selenographic location on the surface. We show a map of all 4902 data points in Fig. 5, with surface crustal field strength contours from an LP ER map constructed using data from the quiet lunar plasma wake and terrestrial magnetotail lobe regions (Mitchell et al., 2008). Recent simulations show that this map primarily represents crustal magnetization with spatial wavelengths greater than
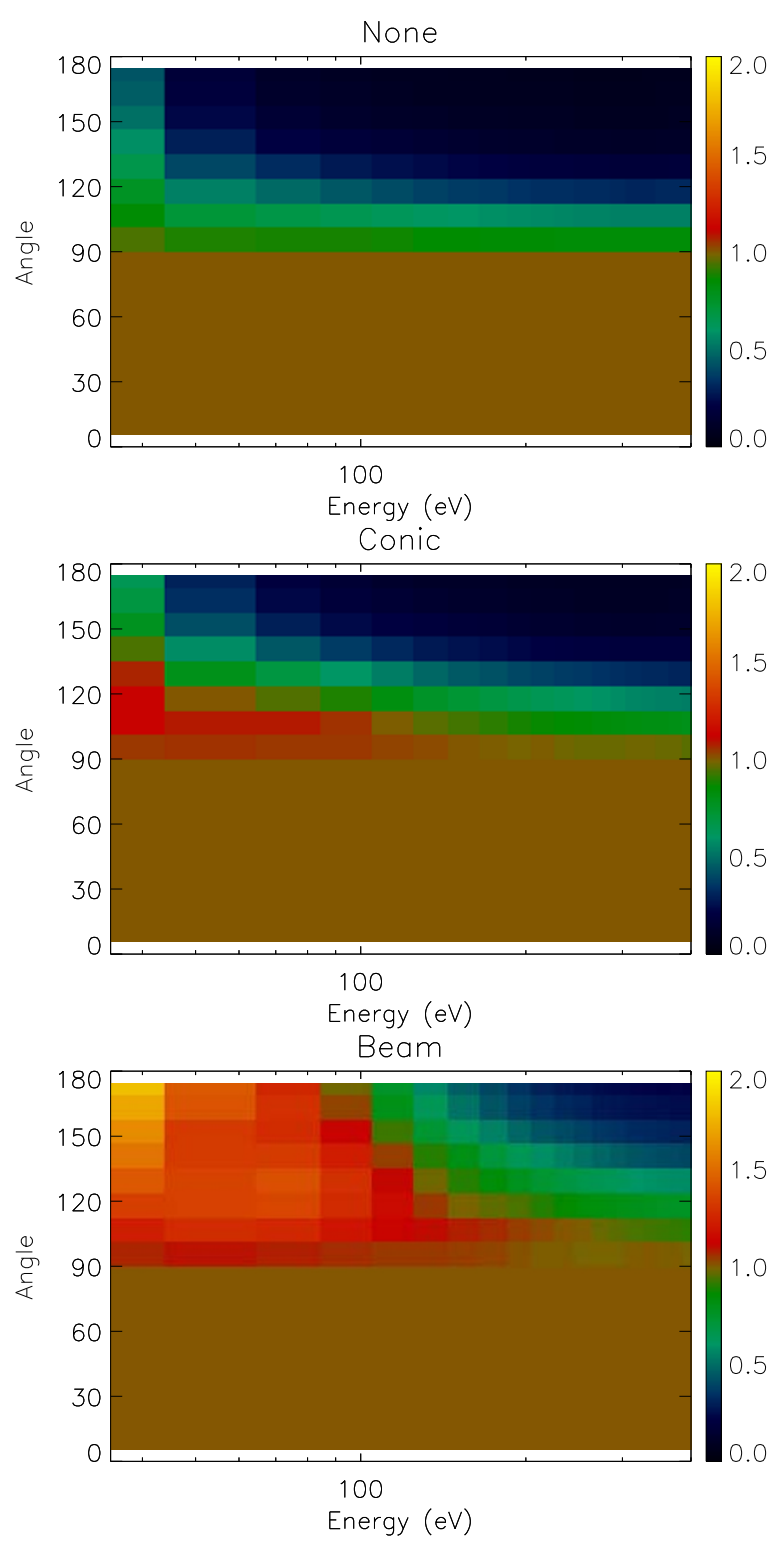

Fig. 4. Median energy-angle distributions for the same three populations in Fig. 3, in the deHoffman-Teller frame, normalized in the same fashion as in Fig. 3.

ten $\mathrm{km}$ (Halekas et al., 2010a). Most dayside solar wind observations lie on the far side of the Moon, because of the phase-locked nature of the lunar orbit around the Earth. We see some time dependent effects in this plot, in the form of track-aligned groupings of similar signatures (e.g. at 235 degrees longitude). However, the form of the measured electron distribution clearly also depends strongly on the crustal magnetic field below the spacecraft, with apparent conics most often observed around the periphery of strong crustal fields, and beams most often observed in stronger magnetic field regions, albeit with significant intermingling of populations. Gaps over the strongest magnetic anomalies result from the data selection described in Section 2.2.

\subsection{Solar wind properties}

We investigated upstream solar wind parameters, including density, temperature, velocity, etc. using Wind measurements (time-shifted appropriately), as well as the magnetic field orientation relative to both the solar wind velocity and 


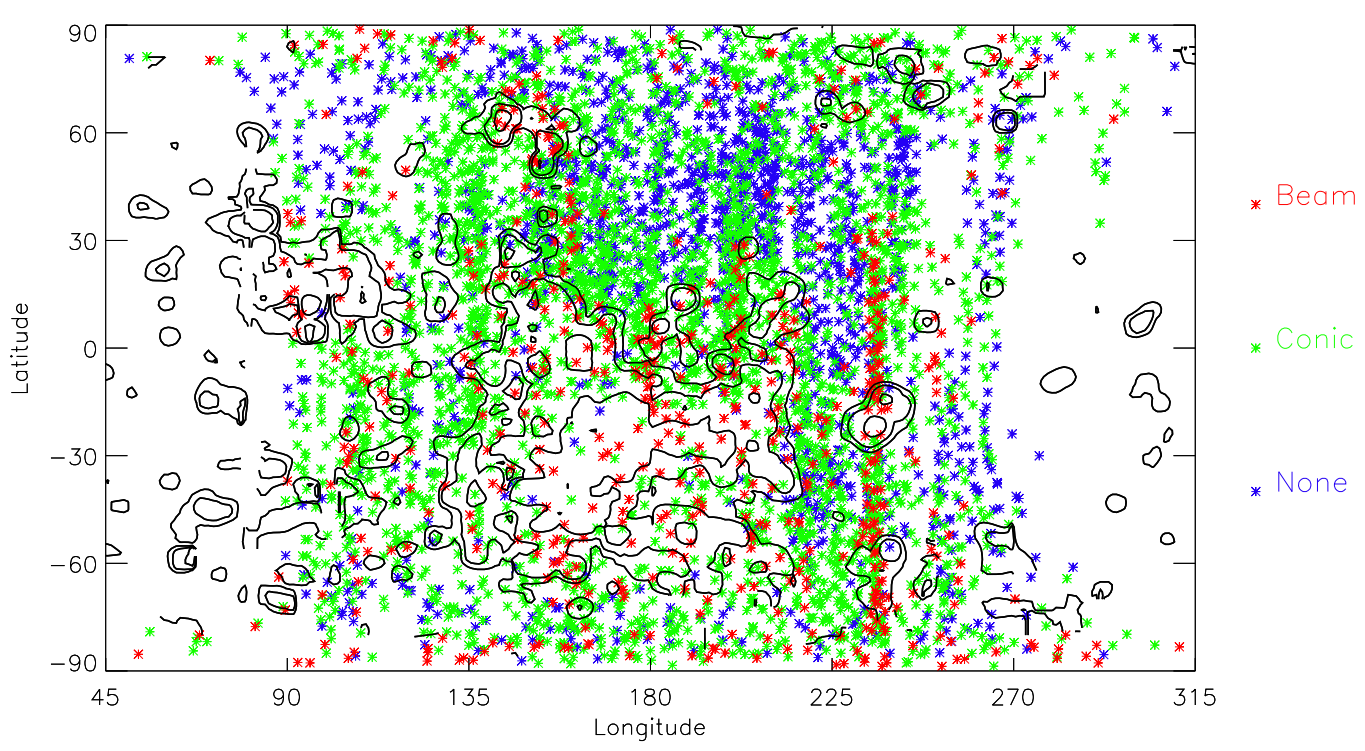

Fig. 5. Selenographic map of the foot-point locations of electron observations (same data set as Figs. 1-3). Blue points indicate observations with no upward-going flux enhancement, green points indicate observations with apparent upward-going conics, and red points indicate observations with upward-going beams. Black contours show $10 \mathrm{nT}, 20 \mathrm{nT}$, and $50 \mathrm{nT}$ levels of surface crustal magnetic field magnitude.

local surface normal, and found no statistically significant differences in the distributions of any solar wind parameter between different types of events, or between events with different loss cone characteristics. This provides evidence that the selenographic location on the surface (in particular, the crustal magnetic field) more strongly influences the form of the electron distribution above the surface than do upstream solar wind parameters. In turn, this also suggests that any differences in downward-going flux (most notably the isotropized suprathermal solar wind flux observed during beam events) most likely result from lunar rather than upstream influences. This lack of sensitivity to solar wind parameters seems inconsistent with simulation results, which predict that electron temperature plays a key role in electron reflection above a surface (Poppe et al., 2011); however, the referenced simulations do not include the effects of crustal magnetic fields.

\section{Electron Reflection and Acceleration Mecha- nisms}

\subsection{Reflection from a moving obstacle}

The comparison between electron distributions in different reference frames in Figs. 1-4 clearly indicates that a significant portion of the energy dependence in the upwardgoing electron population results from reflection of solar wind flux from a moving obstacle. This essentially constitutes a Fermi process, whereby solar wind electrons gain energy upon reflecting from a moving obstacle. This results in a parallel translation of the reflected electron distribution in velocity space. However, while this effect partially explains the observed energy dependent reflection, it certainly does not remove the need for a reflection process, since we can only observe this energy gain if some mechanism operates to reflect the electrons. In addition, in order to successfully explain the majority of our observations, which show energy dependence in all frames, the reflection process must still itself have some intrinsic energy dependence.

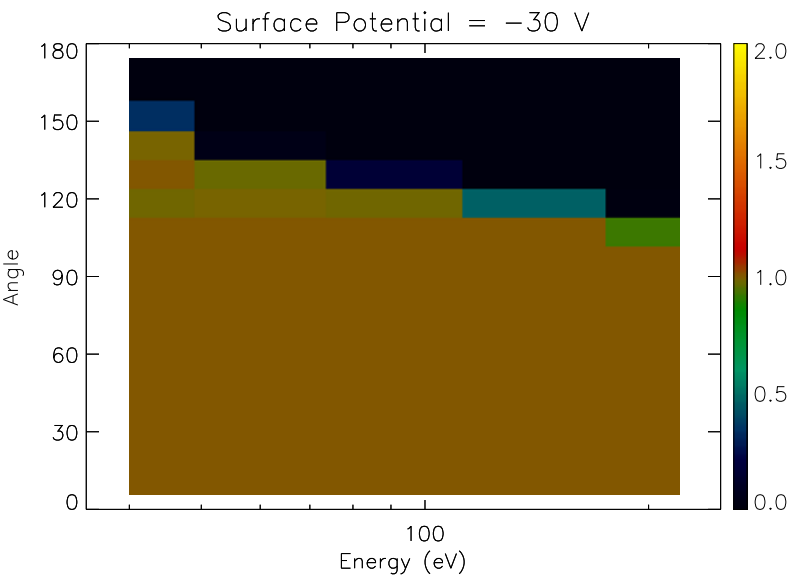

Fig. 6. Model electron energy angle distribution from single-particle tracing simulation with a surface electrostatic potential of $-30 \mathrm{~V}$ and no crustal magnetic fields. Note slightly different energy range $(35-250 \mathrm{eV})$ from measured distributions in Figs. 3 and 4.

\subsection{Electrostatic reflection}

Electrostatic reflection provides a natural candidate to produce the energy dependent reflection we observe. Indeed, we identify an energy dependent loss cone as a consistently observed feature of virtually all dayside solar wind electron observations, in all frames. Such a loss cone generally indicates the presence of a negative potential below the spacecraft (in other words, a downward electric field), either at the surface or between the spacecraft and the surface. We have used single particle tracing simulations (developed to calibrate ER measurements of crustal magnetic fields (Halekas et al., 2010a)) to trace electrons above a negative near-surface potential, and calculate the resulting loss cone distribution. The model electron distribution, shown in Fig. 6, has a loss cone form that closely agrees with the observations. The form of the modeled loss cone distribution proves rather insensitive to the shape or location of the 
negative potential below the spacecraft, with only the total potential drop determining the loss cone. Several possibilities for the source of this potential drop exist.

First, solar wind interaction with crustal magnetic fields may generate electric fields. One expects a charge separation electric field to form in magnetic anomaly regions due to the fact that ions, with larger momentum and gyro-radius, penetrate more easily into crustal field regions than electrons, thus producing an electric field that excludes ions and pulls electrons into the interaction region. Surface experiments (Neugebauer et al., 1972; Goldstein, 1974; Clay et al., 1975) and recent spacecraft measurements (Saito et al., 2010) provide evidence for such charge separation fields. Unfortunately, this electric field should point upward, in the wrong direction to explain our observations. Therefore, while crustal magnetic fields almost certainly play a role, especially given the spatial distribution of the observations and their clear relation to crustal fields, the mechanism seemingly must differ from our basic expectations.

Similar energy dependent loss cones above the lunar night side generally indicate potentials produced by negative surface charging (Halekas et al., 2008c). In this case, one usually observes the energy dependent loss cone at the same time as an upward-going beam, composed of secondary electrons accelerated from the surface. Though the dayside observations do not always show such a beam, the short Debye length $(\sim 10 \mathrm{~m})$ on the dayside (as compared to spacecraft altitude of $\sim 100 \mathrm{~km}$ ) implies that streaming instabilities should have time to develop and smooth out any beam distribution before it reaches the spacecraft, so this does not pose a critical objection. However, given basic expectations from probe theory (Manka, 1973), photoemission should dominate dayside surface charging, forcing the surface to a small positive potential, which again produces an electric field with the wrong sign to explain our observations.

The presence of non-monotonic potentials may provide one escape from this seeming conundrum. Theoretical work (Guernsey and Fu, 1970; Nitter et al., 1998) and recent simulations (Poppe and Horányi, 2010) indicate that the favored electrostatic potential distribution above a photoemitting surface has a potential minimum just above the surface, with most photoelectrons trapped below this minimum, and most incoming electrons reflected above it. The depth of this potential minimum varies linearly with electron temperature. Recent work shows that this mechanism provides a very plausible explanation for inferred negative dayside surface potentials in the terrestrial plasmasheet (Halekas et al., 2008c; Poppe et al., 2011), so it may very likely also operate in the solar wind.

At first glance, a non-monotonic potential structure above the dayside surface seems implausible, but several intuitive explanations exist. First, consider the interface between the near-surface photoelectron sheath, which contains a cold electron population, and the solar wind, which contains a warmer incoming population. This physical situation, with two populations with unequal temperatures in contact, favors the formation of a double layer, with a potential drop on the order of the electron temperature difference (Ishiguro et al., 1985). The superposition of this new double layer with the original sheath naturally results in a non-monotonic potential. In addition, the non-monotonic potential helps to maintain quasi-neutrality upstream from the Moon. As an interplanetary magnetic field line contacts the dayside lunar surface, sunward-going electrons rapidly vacate the field line (because the Moon blocks additional sunward-going electrons from refilling these flux tubes), leaving only a portion of the solar wind electron distribution on the field lines upstream from the Moon. This population cannot balance the charge of the solar wind ion beam. The non-monotonic potential provides a resolution to this situation, by reflecting most of the incoming solar wind electrons, and accelerating enough photoelectrons to the electron temperature to balance any remaining deficit, thus much more closely maintaining quasi-neutrality upstream.

Unfortunately, even a non-monotonic potential, though it does provide an electric field with the right sign, may not prove capable of explaining all of our observations. The expected scale of the potential drop associated with this structure, on the order of the electron temperature, may prove insufficient to explain most of the observations. The inferred potential drop $(\sim 10-100 \mathrm{~V})$ exceeds the electron temperature $(\sim 5-25 \mathrm{eV})$ significantly for many observations. In addition, the potential drop inferred from the electron loss cone does not clearly correlate with solar wind electron temperature (or any solar wind parameters, as discussed in Section 2.5), casting some doubt on this interpretation. Both of these seeming discrepancies may result at least partially from the transformation of electric fields between different reference frames. As with electric fields from magnetic anomalies, the surface field almost certainly plays a role, but it may not explain all of the observations.

Finally, time dependent electric fields may play a role in the electron dynamics. However, the form of these fields, or the mechanism by which they would so consistently produce an energy-dependent loss cone consistent with adiabatic reflection in an electrostatic potential, remains elusive.

\subsection{Non-adiabatic reflection}

As an alternative to adiabatic electrostatic reflection, we considered the possibility of non-adiabatic magnetic reflection. Previous particle tracing simulations have indicated that electrons reflect less efficiently from crustal magnetic fields with wavelengths smaller than the electron gyroradius (Halekas et al., 2010a). This mechanism could thus plausibly form an energy-dependent loss cone, given significant small-scale lunar crustal magnetization. To test this theory, we performed particle-tracing simulations with incoherent crustal magnetization with a wavelength of $4 \mathrm{~km}$ and various average field strengths at the surface, ranging from 1-50 nT. The results in Fig. 7 indicate that such incoherent small-scale crustal fields can indeed produce energy-dependent electron reflection. However, the reflected population, in general, does not have as much flux as the downward-going population. Instead, the reflection appears more diffuse in form, consistent with the stochastic and gyrophase-dependent nature of reflection in such inhomogeneous magnetic fields. Furthermore, this process requires rather strong crustal fields to produce significant energy-dependent electron reflection. Thus, we conclude that the non-adiabatic magnetic reflection mech- 

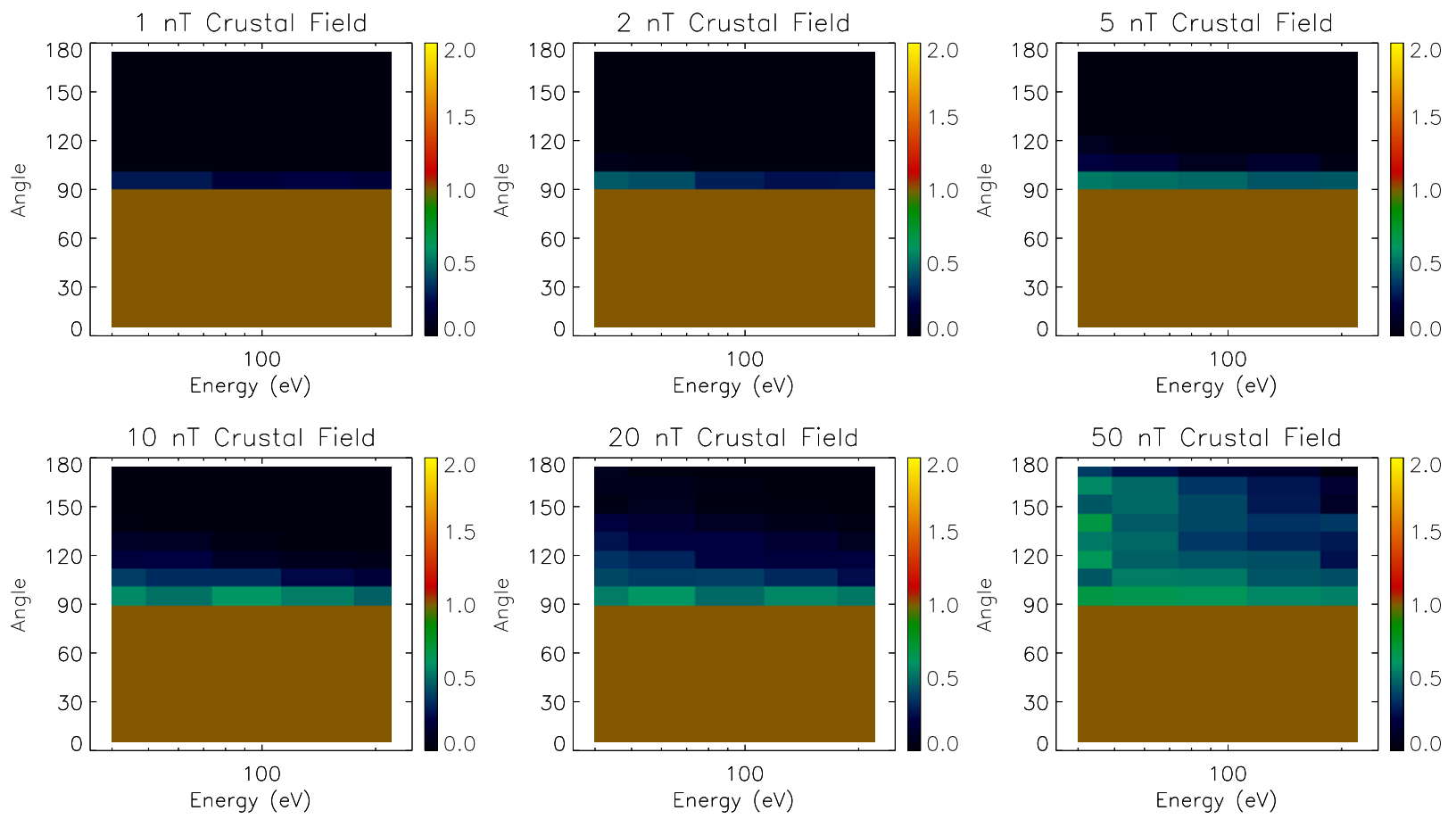

Fig. 7. Model electron energy angle distributions from particle tracing simulations with zero surface potential and incoherent crustal magnetization with $4 \mathrm{~km}$ wavelength and strength sufficient to produce average surface crustal field magnitudes of 1, 2, 5, 10, 20, and 50 nT. Note slightly different energy range (35-250 eV) from measured distributions in Figs. 3 and 4.

anism, though it may play a role, cannot alone explain our data.

Other non-adiabatic effects resulting from the small spatial scale of electric field layers could also prove important. For instance, previous studies have shown that suprathermal electrons reflect less efficiently from thin surface electric field layers when the IMF intersects the surface at an oblique angle (Halekas et al., 2003). Just such cases also require the largest transformation velocities between the dHT frame and other frames, suggesting the possibility of unexpected effects in this regime.

\subsection{Conic formation}

In addition to energy dependent electron reflection, we must also explain the observation of apparent upward-going electron conics. As we showed in Section 2.3, these conics primarily result from the energy gain from reflection from a moving surface. However, some weak conic signatures remain even in the $\mathrm{dHT}$ frame (though these could conceivably result from frame transformation errors or nonadiabatic effects). In addition, beam distributions have a somewhat conic form in the $\mathrm{dHT}$ frame. This may simply result from parallel relaxation of an upward-going beam as a result of streaming instabilities. However, it could also result from some heating mechanism and/or an additional electron population.

The observed conics seemingly cannot form by adiabatic reflection of a beamed source distribution with a width larger than the loss cone, as seen sometimes at Earth (Burch, 1995), because the upward-going flux at low energies exceeds the corresponding downward-going flux at oblique angles. Nor can we explain all observations by invoking pitch angle scattering that broadens an anisotropic source distribution that then reflects, thus forming a conic distribu- tion. At low energies, the total observed integrated upwardgoing fluxes often exceed the total integrated downwardgoing fluxes, which a pitch angle scattering mechanism alone cannot explain (though it could play a role).

Furthermore, we cannot easily explain the observed conics as secondary electrons and/or photoelectrons from the surface. If not accelerated, these electrons would have too little energy. If accelerated, on the other hand, they should form beamed distributions, or possibly conics with an angle related to the angle between the magnetic field and the surface normal (Halekas et al., 2010b). The conic angles seen in the observations do not obviously conform to this latter expectation; however, the magnetic field angle at the surface could differ significantly from that observed at spacecraft altitude, so we cannot rule out this possibility.

Other conic formation mechanisms include parallel heating/acceleration by time-varying electric fields (André and Eliasson, 1992) or other stochastic mechanisms (Temerin and Cravens, 1990), and perpendicular wave heating (Menietti and Burch, 1985; Roth et al., 1989). Parallel heating alone probably cannot explain the observed conics, given the enhanced perpendicular flux (Temerin and Cravens, 1990). One interesting possibility is a perpendicular heating mechanism identified by Roth et al. (1989), consisting of a loss cone instability that excites upper hybrid waves, which in turn heat electrons in the perpendicular direction. This mechanism has a number of attractive features. First the mechanism requires both a warm electron population with a loss cone distribution, and a cold electron distribution. At the Moon, the incoming solar wind distribution naturally provides the former, and the component of photoelectrons and/or secondary electrons that escapes from the near-surface sheath region naturally provides the 
latter. The predicted heating resulting from this process should be efficient at parallel electron velocities a few times the thermal speed of the warm population, or in our case a few tens of $\mathrm{eV}$, in relatively good agreement with our observations. Furthermore, this model naturally provides for different efficiencies of conic generation above crustal magnetic fields, since the mechanism will likely vary in efficiency with the size of the loss cone, and with the ratio of escaping cold flux from the surface to incident and/or reflected solar wind flux.

\subsection{Beam acceleration}

Finally, to generate the more rarely seen beam distributions (12\% of observations), a number of mechanisms may play a role. If electrostatic fields indeed prove responsible for the observed energy dependent electron reflection, these fields will also accelerate secondary electrons and/or photoelectrons from the surface, producing an upward-going beam. This beam should prove unstable to various streaming instabilities, naturally spreading it in energy and angle, commensurate with observations. These instabilities would also affect the incoming population, possibly leading to the isotropized downward-going flux that we observe for beam distributions, either directly through a streaming instability, or via production of whistlers that could then cause pitch angle diffusion.

However, time-varying electric fields could also play a role in beam production and electron heating. Solar wind interaction with magnetic anomalies could produce such fields, perhaps via streaming instabilities resulting from ion reflection in the anomaly regions. In this case, the waves thereby set up should also affect solar wind ions, with the likely result of converting ion flow energy into thermal energy. Preliminary indications from Kaguya observations suggest that one can indeed observe such changes in the solar wind ion distribution at low altitudes in magnetic anomaly regions (Saito et al., private communication).

\section{Implications}

The observations presented in this paper may have a number of implications for other aspects of the lunar environment, depending on the mechanism(s) responsible. The likely presence of downward electric fields at or near the surface has implications for sputtered and exospheric ions, as well as dust. Any ions produced in this field region will feel a downward force, possibly enough to trap and/or drive ions back into the surface. In the case of a non-monotonic near-surface potential, the resulting potential minimum could even locally trap some photo-ions. Similarly, electric fields will affect any dust grains that have left the surface as a result of electrostatic forces or micrometeorite bombardment. In the case of a non-monotonic plasma sheath, non-neutral plasma layers could also affect the charging of these dust grains.

The various wave modes considered in this paper may also interact with incoming solar wind electrons and ions, or with charged particles produced at the surface, in unexpected fashions. Indeed, we have presented evidence that upward-going electron beams sometimes observed near strong crustal fields may interact with incoming electrons, producing instabilities and isotropizing the suprathermal solar wind electron population.

In fact, our observations suggest that a precursor layer consisting of a standing electrostatic structure, associated with crustal magnetic anomalies and/or non-monotonic near-surface potentials, may exist above the lunar surface in the solar wind. A portion of the incoming solar wind electrons reflect from this precursor layer, gaining additional energy during the reflection process as a result of the motion of the Moon relative to the solar wind. Meanwhile, a portion of the photoelectron distribution generated at the surface will escape from this layer. Both reflected and locally produced electrons stream outward into the solar wind plasma, helping to ensure quasi-neutrality on flux tubes that connect to the lunar surface, and very likely interacting with and perturbing solar wind electrons upstream from the Moon.

The instabilities produced by the effects of this precursor layer might measurably perturb incoming solar wind ions as well as electrons. In addition, as recent measurements from Kaguya and Chandrayaan show, a significant fraction of ions reflect from the lunar surface and crustal magnetic fields; these reflected ions should also affect the incoming solar wind ion and electron populations. Therefore, a growing body of evidence suggests that the Moon's presence may affect the incoming solar wind plasma at larger distances upstream than often considered.

Acknowledgments. We thank NASA's Lunar Science institute for supporting this work.

Guest editor M. Yamauchi thanks two anonymous reviewers in evaluating this paper.

\section{References}

André, M. and L. Eliasson, Electron acceleration by low frequency electric field fluctuations: Electron conics, Geophys. Res. Lett., 19, 1073-1076, 1992.

Burch, J. L., Dynamics Explorer observations of the production of electron conics, Geophys. Res. Lett., 22, 2705-2708, 1995.

Clay, D. R., B. E. Goldstein, M. Neugebauer, and C. W. Snyder, Lunar surface solar wind observations at the Apollo 12 and Apollo 15 sites, $J$. Geophys. Res., 80, 1751-1760, 1975.

deHoffman, F. and E. Teller, Magnetohydrodynamic shocks, Phys. Rev., 80, 692, 1950.

Feuerbacher, B., M. Anderegg, B. Fitton, L. D. Laude, R. F. Willis, and R. J. L. Grard, Photoemission from lunar surface fines and the lunar photoelectron sheath, Proc. Lunar Sci. Conf., 3, 2655-2663, 1972.

Fitzenreiter, R. J., J. D. Scudder, and A. J. Klimas, Three-dimensional analytical model for the spatial variation of the foreshock electron distribution function: Systematics and comparisons with ISEE observations, J. Geophys. Res., 95, 4155-4173, 1990.

Freeman, J. W. and M. Ibrahim, Lunar electric fields, surface potential and associated plasma sheaths, Moon, 14, 103-114, 1975.

Goldstein, B. E., Observations of electrons at the lunar surface, J. Geophys. Res., 79, 23-35, 1974.

Guernsey, R. L. and J. H. M. Fu, Potential distribution surrounding a photoemitting plate in a dilute plasma, J. Geophys. Res., 75(16), 3193-3199, 1970.

Halekas, J. S., R. P. Lin, and D. L. Mitchell, Inferring the scale height of the lunar nightside double layer, Geophys. Res. Lett., 30, 2117 , doi:10.1029/2003GL018421, 2003.

Halekas, J. S., D. A. Brain, D. L. Mitchell, and R. P. Lin, Whistler waves observed near lunar crustal magnetic sources, Geophys. Res. Lett., 33, L22104, doi:10.1029/2006GL027684, 2006.

Halekas, J. S., G. T. Delory, D. A. Brain, R. P. Lin, and D. L. Mitchell, Density cavity observed over a strong lunar crustal magnetic anomaly in the solar wind: A mini-magnetosphere?, Planet. Space Sci., 56(7), 941-946, doi:10.1016/j.pss.2008.01.008, 2008a.

Halekas, J. S., D. A. Brain, R. P. Lin, and D. L. Mitchell, Solar wind interaction with lunar crustal magnetic anomalies, J. Adv. Space Res., 
41, 1319-1324, doi:10.1016/j.asr.2007.04.003, 2008b

Halekas, J. S., G. T. Delory, R. P. Lin, T. J. Stubbs, and W. M. Farrell, Lunar Prospector observations of the electrostatic potential of the lunar surface and its response to incident currents, J. Geophys. Res., 113, A09102, doi:10.1029/2008JA013194, 2008c.

Halekas, J. S., R. J. Lillis, R. P. Lin, M. Manga, M. E. Purucker, and R. A. Carley, How strong are lunar crustal magnetic fields at the surface: Considerations from a reexamination of the electron reflectometry technique, J. Geophys. Res., 115, E03006, doi:10.1029/2009JE003516, 2010a

Halekas, J. S., Y. Saito, G. T. Delory, and W. M. Farrell, New views of the lunar plasma environment, Planet. Space Sci., doi:10.1016/j.pss.2010.08.011, 2010b (in press).

Horányi, M., B. Walch, S. Robertson, and D. Alexander, Electrostatic charging properties of Apollo 17 lunar dust, J. Geophys. Res., 103, 8575-8580, 1998

Ishiguro, S., T. Kamimura, and T. Sato, Double layer formation caused by contact between different temperature plasmas, Phys. Fluids, 28, 2100$2105,1985$.

Lin, R. P., D. L. Mitchell, D. W. Curtis, K. A. Anderson, C. W. Carlson, J. McFadden, M. H. Acuña, L. L. Hood, and A. Binder, Lunar surface magnetic fields and their interaction with the solar wind: Results from Lunar Prospector, Science, 281, 1480-1484, 1998.

Manka, R. H., Plasma and potential at the lunar surface, in Photon and Particle Interactions with Surfaces in Space, edited by Grard, R. J. L. and D. Reidel, pp. 347-361, Dordrecht, Netherlands, 1973.

McComas, D. J., F. Allegrini, P. Bochsler, P. Frisch, H. O. Funsten, M. Gruntman, P. H. Janzen, H. Kucharek, E. Möbius, D. B. Reisenfeld, and N. A. Schwadron, Lunar backscatter and neutralization of the solar wind: First observations of neutral atoms from the Moon, Geophys. Res. Lett., 36, L12104, doi:101029/2009GL038794, 2009.

Menietti, J. D. and J. L. Burch, Electron conic signature observed in the nightside auroral zone and over the polar cap, J. Geophys. Res., 90, 5345, 1985.

Mitchell, D. L., J. S. Halekas, R. P. Lin, S. Frey, L. L. Hood, M. H. Acuna, and A. Binder, Global mapping of lunar crustal magnetic fields by $\mathrm{Lu}-$ nar Prospector, Icarus, 194, 401-409, doi:10.1016/j.icarus.2007.10.027, 2008.

Neugebauer, M., C. W. Snyder, D. R. Clay, and B. E. Goldstein, Solar wind observations on the lunar surface with the Apollo 12 ALSEP, Planet. Space Sci., 20, 1577-1591, 1972.

Nitter, T., O. Havnes, and F. Melands $\emptyset$, Levitation and dynamics of charged dust in the photoelectron sheath above surfaces in space, J. Geophys. Res., 103, 6605-6620, 1998.

Poppe, A. and M. Horányi, Simulations of the photoelectron sheath and dust levitation on the lunar surface, J. Geophys. Res., 115, A08106,
doi:10.1029/2010JA015286, 2010.

Poppe, A., J. S. Halekas, and M. Horányi, Negative potentials above the day-side lunar surface in the terrestrial plasma sheet: Evidence of nonmonotonic potentials, Geophys. Res. Lett., doi:10.1029/2010GL046119, 2011 (in press).

Roth, I., M. K. Hudson, and M. Temerin, Generation models of electron conics, J. Geophys. Res., 94, 10,095-10,102, 1989.

Saito, Y., S. Yokota, T. Tanaka, K. Asamura, M. N. Nishino, M. Fujimoto, H. Tsunakawa, H. Shibuya, M. Matsushima, H. Shimizu, F. Takahashi, T. Mukai, and T. Terasawa, Solar wind proton reflection at the lunar surface: Low energy ion measurements by MAPPACE onboard SELENE (KAGUYA), Geophys. Res. Lett., 35, L24205, doi:10.1029/2008GL036077, 2008.

Saito, Y., S. Yokota, K. Asamura, T. Tanaka, M. N. Nishino, T. Yamamoto, Y. Terakawa, M. Fujimoto, H. Hasegawa, H. Hayakawa, M. Hirahara, M. Hoshino, S. Machida, T. Mukai, T. Nagai, T. Nagatsuma, T. Nakagawa, M. Nakamura, K. Oyama, E. Sagawa, S. Sasaki, K. Seki, I. Shinohara, T. Terasawa, H. Tsunakawa, H. Shibuya, M. Matsushima, H. Shimizu, and F. Takahashi, In-flight performance and initial results of Plasma energy Angle and Composition Experiment (PACE) on SELENE (Kaguya), Space Sci. Rev., doi:10.1007/s11214-010-9647-x, 2010.

Temerin, M. A. and D. Cravens, Production of electron conics by stochastic acceleration parallel to the magnetic field, J. Geophys. Res., 95, 4285-4290, 1990.

Whipple, E. C., Potentials of surfaces in space, Rep.Prog.Phys., 44, $1197-$ 1250, 1981.

Wieser, M., S. Barabash, Y. Futaana, M. Holmstrom, A. Bhardwaj, R. Sridharan, M. B. Dhanya, P. Wurz, A. Schaufelberger, and K. Asamura, Extremely high reflection of solar wind protons as neutral hydrogen atoms from regolith in space, Planet. Space Sci., doi:10.1016/j.pss.2009.09.012, 2009.

Wieser, M., S. Barabash, Y. Futaana, M. Holmstrom, A. Bhardwaj, R. Sridharan, M. B. Dhanya, P. Wurz, A. Schaufelberger, and K. Asamura, First observation of a mini-magnetosphere above a lunar magnetic anomaly using energetic neutral atoms, Geophys. Res. Lett., 37, L015103, doi:10.1029/2009GL041721, 2010.

Willis, R. F., M. Anderegg, B. Feuerbacher, and B. Fitton, Photoemission and secondary electron emission from lunar surface material, in Photon and Particle Interactions with Surfaces in Space, edited by Grard, R. J. L. and D. Reidel, pp. 389-401, 1973.

J. S. Halekas (e-mail: jazzman@ssl.berkeley.edu), A. Poppe, G. T. Delory, W. M. Farrell, and M. Horányi 